\title{
Observations on glutaraldehyde-treated heterologous cardiac valves
}

\author{
E BODNAR, ${ }^{1}$ E G J OLSEN, ${ }^{2}$ AND D N ROSS ${ }^{1}$
}

From the Department of Surgery, Cardiothoracic Institute, ${ }^{1}$ and the Department of Histopathology, National Heart Hospital, ${ }^{2}$ London W1, UK

ABSTRACT Recent publications on commercial porcine bioprosthetic cardiac valves have stated that the endothelium disappears during the glutaraldehyde processing and that in many cases histological evidence of early tissue deterioration was found. Our experiences have shown that porcine aortic valves can be processed in glutaraldehyde without loss of the integrity of the endothelium and with the development of optimum collagen fibre structure. The valve mounted in a Dacron tube is not restricted in diameter and performs well under biological conditions up to a period of six months.

Bioprosthetic cardiac valves in clinical use are processed in glutaraldehyde at one stage of preparation, and these valves are thought to be stable and biologically inert, both these advantages being attributed to the glutaraldehyde (Carpentier et al, 1969; Zuhdi, 1976; Ionescu et al, 1977; Angell et al, 1977). The length of the collagen cross-links formed by this four carbon atom open chain aldehyde proved favourable for the durability of a cardiac bioprosthetic valve, and probably glutaraldehyde eliminates the antigenicity of the heterologous tissue. Bioprosthetic valves are far less thrombogenic than prosthetic ones (Oyer et al, 1977). Nevertheless, there are justifiable reservations against their exclusive use. The anatomical structure of the pig aortic valve makes it more or less restrictive so that it causes functional valvar stenosis (Johnson et al, 1976; Lurie et al, 1976). Furthermore, there is increasing pathological evidence questioning the inertness of the glutaraldehyde-processed tissue under biological conditions (Spray et al, 1976; Fishbein et al, 1977; Spray and Roberts, 1977; Ashraf and Bloor, 1978). Recently cellular damage in the valve cusps caused by the standard, commercial glutaraldehyde processing has been described (Ferrans et al, 1978).

The increasing number of publications criticising the haemodynamic performance as well as the morphological integrity of the glutaraldehydetreated porcine aortic valve prcmpted us to report our experiences in this field.

\section{Materials and methods}

One hundred and fifty-seven porcine aortic and pulmonary valves were selected for the study. The $\stackrel{\varnothing}{\circledR}$ hearts were delivered to the laboratory within six $\vec{F}$ hours of slaughter. The valves were immediately $\frac{9}{3}$ dissected, immersed in Hartman's solution at $4^{\circ} \mathrm{C}$, and washed for 12 hours. Four per cent formaldehyde or $0.1 \%$ glutaraldehyde was used as fixative. Five different methods of fixation were compared 으 as follows: formaldehyde or $0.1 \%$ glutaraldehyde $\underset{\times}{\otimes}$ with the valve left intact, $0 \cdot 1 \%$ glutaraldehyde with valve cusps packed with cotton wool, $0.1 \%$ glutaraldehyde applied with $100 \mathrm{mmHg}$ constant $\delta$ pressure on unsupported valves, and, finally, the same method, but with the valves encapsulated within a Dacron tube. For animal experiments the $D$ conduits were sterilised in $2 \%$ glutaraldehyde을. solution (Cidex, Ethicon Ltd).

Tissue samples were obtained at one day, one ${ }^{\circ}$ week, and one, three, and six months after the ${ }_{0}$ start of fixation. The tissue was cut into suitable $\mathbb{W}^{N}$ blocks that were paraffin embedded. The $5 \mu \mathrm{go}$ thick sections were cut and stained with haemaro toxylin and eosin and Weigert's elastic Van Gieson. In cases of fibrin deposition Marshall's? scarlet blue (Lendrum et al, 1962) was used.

Seven mongrel dogs with a bodyweight of 20-울 $25 \mathrm{~kg}$ were operated on and allowed to survive for six months. Under intratracheal halothane $\stackrel{\mathbb{Q}}{\stackrel{\mathbb{Q}}{2}}$ anaesthesia the left chest was opened and a lefto ventricular-aortic conduit containing a biopros- $\Omega$ 
thetic valve inserted without the use of cardiopulmonary bypass. A direct contact was created between host epicardium and myocardium and implanted valve as the valves were placed at the cardiac end of the conduit. No anticoagulants were used during the whole experimental period apart from a large dose of heparin immediately before the death of the animals.

Before death the animals were anaesthetised and ventilated with room air. Pressures were recorded in the left ventricle, conduit, ascending aorta, and abdominal aorta, and cineangiograms of the left ventricle and conduit were obtained. The heart and conduit were removed for macroscopic, and the valves for macroscopic and microscopic, examination.

\section{Results}

\section{EFFECT OF FIXATION}

Definite differences were observed in the valves subjected to the two methods of fixation. In those fixed in $4 \%$ formaldehyde foci of disarray of collagen tissue and areas of necrosis were found-that is, the fibrillar characteristics of collagen tissue were lost, and the tissue assumed a smudged outline (fig 1). These changes were not seen in any of the glutaraldehyde-treated valves.

\section{EFFECT OF PHYSICAL MANIPULATION}

Valves subjected to a pressure of $100 \mathrm{mmHg}$ were superior to those packed with cotton wool because the normal anatomical configuration of the leaflets as well as the root of the aorta were preserved. In both these methods of approach stretching of leaflets was observed. Microscopically normal architecture was well preserved, and the endothelial cells and the collagen fibres were regularly arranged in the valve leaflets that were of uniform thickness (fig 2). No histological differences could be found in the leaflets packed in cotton wool or subjected to pressure of $100 \mathrm{mmHg}$. The histological appearances did not vary with increased time of storage. The valves were readily accommodated in the Dacron tube. The muscular right coronary cusps caused no stenosis and the coaptation of the three cusps was normal in the closed position (figs $3 a \&$ b).

\section{ANIMAL EXPERIMENTS}

All animals survived the six-month period without clinical signs of cardiac failure. At examination a systolic murmur was heard above the apex and the conduit. The femoral arterial pulse was normal in every animal. Manometric data are summarised in the table.

Cineangiograms from the left ventricle showed simultaneous opacification of the ascending aorta and conduit in five animals with an aortic valve sewn into the Dacron graft (fig 4). The filling of both the pulmonary valve-containing conduits was somewhat delayed. Angiograms taken from these two conduits showed significant reflux into the left ventricle through the pulmonary valves. There was

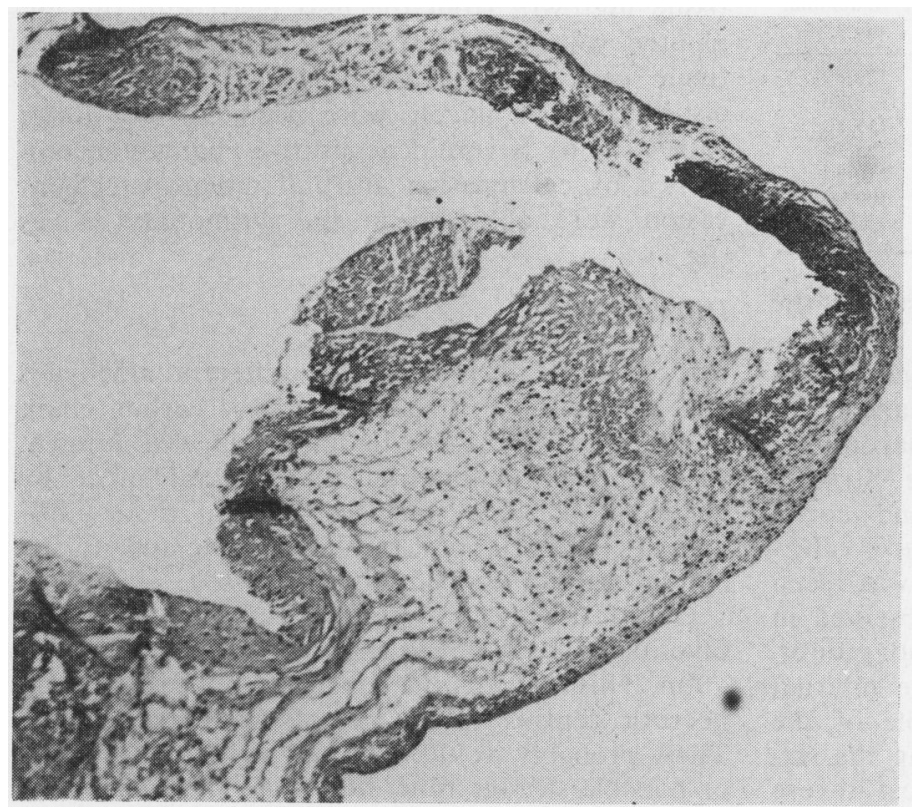

Fig 1 Four per cent formaldehyde fixed valve leaflet showing several foci of necrosis of collagen tissue and a concomitant decrease in number of nuclei (Haematoxylin and eosin $\times 50)$. 


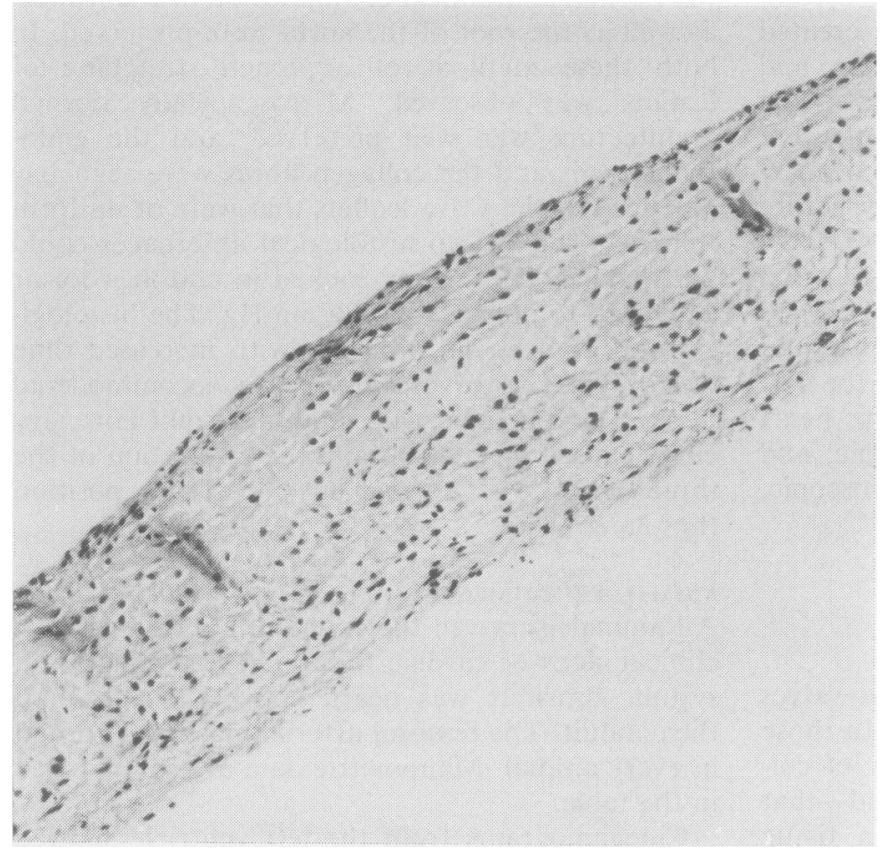

Fig 2 Photomicrograph of an aortic valve cusp which had been subjected to $100 \mathrm{~mm} \mathrm{Hg}$ pressure in $0.1 \%$ glutaraldehyde. Normal architecture is preserved $(H \& E \times 100)$.
Ventriculo-aortic valved conduit: pressures in aorta and left ventricle

\begin{tabular}{lccc}
\hline & \multicolumn{2}{l}{ Pressures $\mathrm{mmHg}$} \\
\cline { 2 - 4 } Conduit & $L V$ & $\begin{array}{c}\text { Ascending } \\
\text { aorta }\end{array}$ & $\begin{array}{c}\text { Abdominal } \\
\text { aorta }\end{array}$ \\
\hline Aortic No 1 & $120 / 0$ & $120 / 80$ & $120 / 80$ \\
Aortic No 2 & $115 / 0$ & $115 / 80$ & $115 / 80$ \\
Aortic No 3 & $120 / 0$ & $120 / 90$ & $115 / 80$ \\
Aortic No 4 & $95 / 0$ & $95 / 70$ & $95 / 70$ \\
Aortic No 5 & $110 / 0$ & $110 / 85$ & $110 / 85$ \\
Pulmonary No 1 & $115 / 0$ & $115 / 70$ & $100 / 80$ \\
Pulmonary No 2 & $120 / 0$ & $120 / 80$ & $100 / 65$ \\
\hline
\end{tabular}

no reflux through the aortic valves inserted in the Dacron graft.

At necropsy no evidence of cusp degeneration was seen in any of the aortic valve leaflets. Ingrowth of fibrous tissue did not extend beyond the level of the glutaraldehyde-treated valve. Striking differences between the pulmonary and aortic valves were observed. In the pulmonary valves shrinkage and obvious calcification was seen whereas none of these changes were observed in the leaflets of the five aortic valves. In both groups, however, calcification was found on the internal surface of the conduit. The apical hole of the left ventricle had become smaller during the sixmonth period of observation. The most severe changes were seen in the two cases with pulmonary valve implantation.

Corresponding with the macroscopic observation, there was no histological evidence of cellular tissue ingrowth from the host heart into the implanted valve (fig 5). Cellular elements of donor $\tilde{x}$ tissue were retained, and only occassional foci of $\dot{0}$ disorientated collagen were seen in the aortic valves (fig 6). Severe degenerative changes accompanied by calcification and inflammatory cell in-

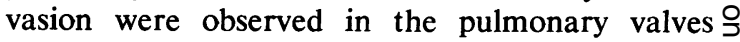
(fig 7).

\section{Discussion}

The effect of fixation of the and its relation to the length of the carbon chain attached to the aldehyde group is well known. Carpentier has pointed out that glutaraldehyde, by

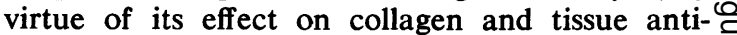
genicity, gives the best results in fixing and storing cardiac valves (Carpentier et al, 1969).

Our observations suggest a third factor which favours glutaraldehyde rather than formalin fix- $\mathbb{D}$ ation. This factor is the consistent presence of tiny $\frac{\stackrel{O}{\mathbb{D}}}{\mathrm{D}}$ necrotic centres in the formaldehyde-fixed tissue. 을 These probably would not alter physical properties or possible storage time, but under biological con- 

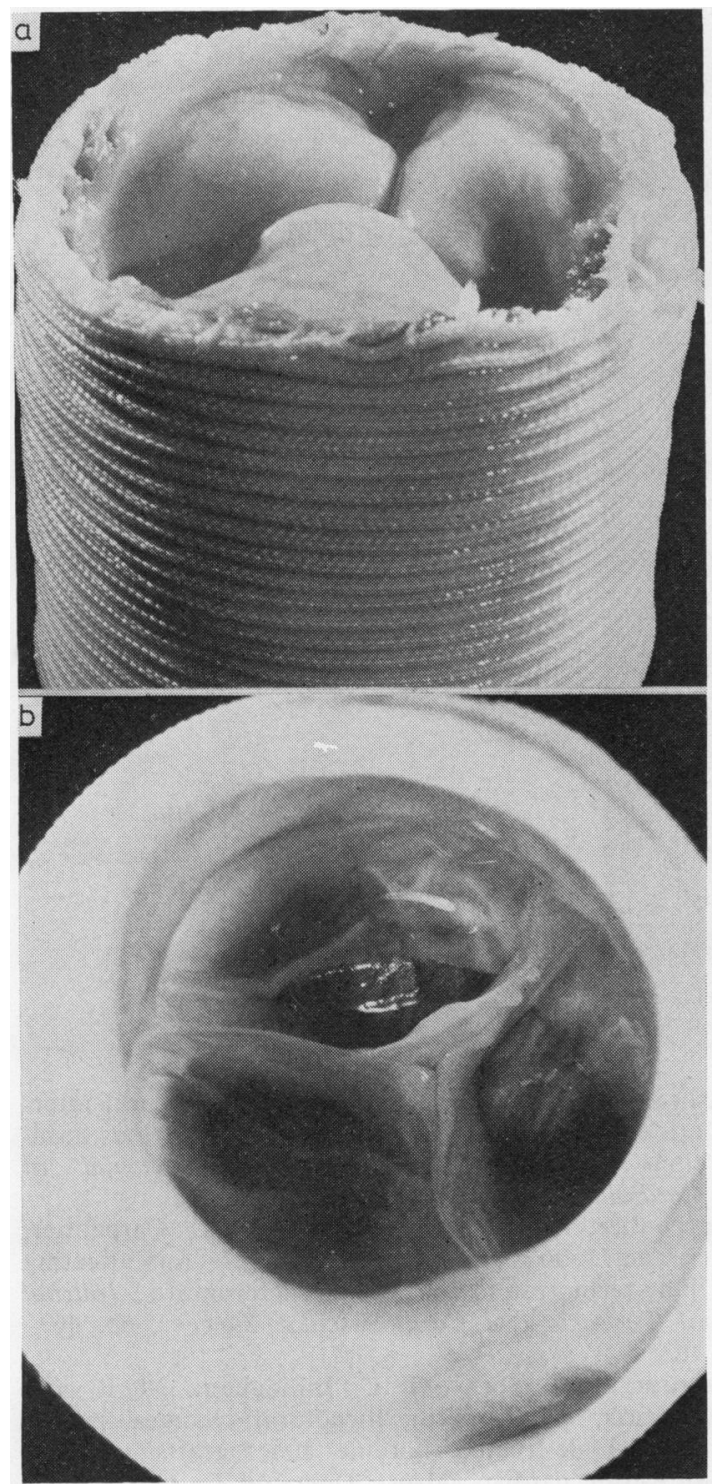

Fig 3a \& b Porcine aortic valve in a Dacron conduit.

ditions they can lead to cellular invasion that induces degeneration followed by late calcification.

The comparison of different methods of handling of the valves during fixation has shown that neither application of a pressure of $100 \mathrm{mmHg}$ nor cotton wool packing of the valve damages the endothelial or fibre structure, as assessed by light microscopic examination. The collagen fibres become well organised and well orientated in the closed position when the valve is fixed under pressure in glutaraldehyde. The accommodation of valves in a Dacron conduit was accomplished with a suture line excluding the muscular part of the right coronary cusp, thus causing no stenosis whatsoever.

In animal experiments the glutaraldehydetreated aortic valve produced excellent overall performance during the six months. None of the valves became either stenotic or incompetent. The extensive scar tissue around the apical ventricular hole did not extend into the glutaraldehyde-fixed heterologous tissue, although there was no mechanical barrier between host and donor biological parts. Although the valves in the Dacron tube were attached directly to the myocardium, thus deliberately creating the possibility of connective tissue ingrowth, only slight and inconsistent cellular infiltration was seen.

Significant differences in location and amount of calcification were observed between aortic and pulmonary valves. The calcium deposits never occurred in the valve leaflets of the aortic valves. The structure of the pulmonary valve cusps was completely destroyed by degeneration and calcification, despite the lack of histological evidence that pulmonary valves would have reacted differently from aortic ones to glutaraldehyde fixation. The degenerated pulmonary valves showed no signs of overstress from the inappropriately high pressure in the left ventricle-aortic position. The probable explanation of the different results lies in the different anatomical shape of the aortic and pulmonary artery roots. The former can be accommodated in the Dacron tube with only the sinuses deformed, but this is impossible in the latter because of the striking difference between the diameter of the pulmonary valvar annulus and that of the main pulmonary artery. We think it is the total deformation of the original anatomical shape that leads to degeneration and, finally, calcification in the pulmonary valves. This observation underlines the importance of the appropriate stream conditions for any given bioprosthesis.

\section{Conclusions}

(1) Histological evidence is presented that $0.1 \%$ glutaraldehyde fixation is superior to $4 \%$ formaldehyde.

(2) Prevention of shrinkage by physical means during fixation in glutaraldehyde has no deleterious effect on tissue structure.

(3) Light microscopy shows that valve leaflets fixed in glutaraldehyde at a pressure of $100 \mathrm{mmHg}$ have optimum collagen fibre structure and the endothelium is left intact.

(4) The collagen and elastic fibres in valve tissue 


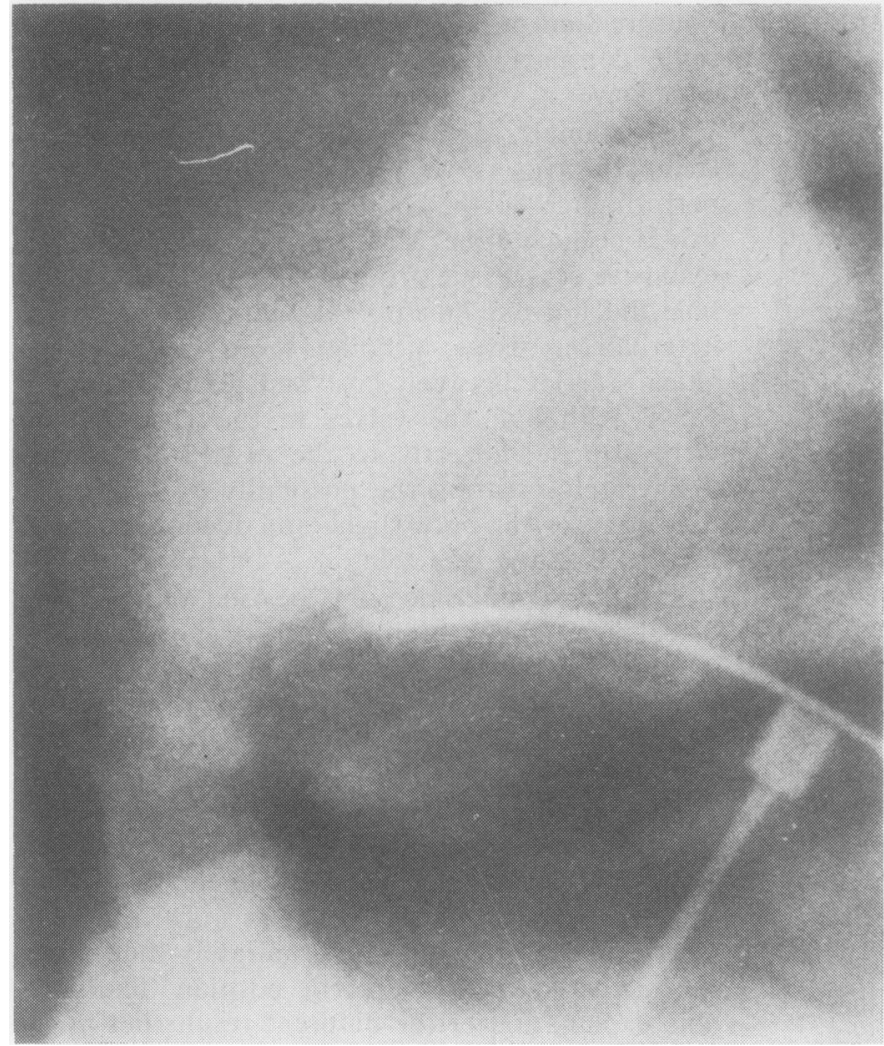

Fig 4 Left ventricular angiogram taken six months after insertion of a left ventricle-aorta valved conduit. Opacification in left ventricle, ascending aorta, and conduit.

once fixed in glutaraldehyde will not change their orientation with increasing duration of preservation up to a period of six months.

(5) Porcine aortic valves treated with glutaraldehyde give excellent haemodynamic performance in dogs over a six-month period.

(6) Glutaraldehyde not only makes the biological tissue inert, but prohibits ingrowth of artificially induced scar tissue.

(7) Mechanical factors, such as stream conditions, can determine degeneration in biological valve prostheses.

We should like to thank Ethicon Ltd for their generous sponsorship of this project and all those at John Sainsbury and Haverhill Meat Products for donating time, interest, and raw materials to the project. Finally, we should like to thank Miss Carol Outram for her technical and secretarial help.

\section{References}

Angell, W W, Angell, J D, and Sywak, A (1977). Selection of tissue or prosthetic valve. Journal of Thoracic and Cardiovascular Surgery, 73, 43-53.
Ashraf, M, and Bloor, C M (1978). Structural alterations of the porcine heterograft after various durations of implantation. American Journal of Cardiology, 41, 1185-1190.

Carpentier, A, LeMaigre, G, Robert, L, Carpentier, $\mathrm{S}$, and Dubost, C (1969). Biological factors affecting 3 long-term results of valvular heterografts. Journal of Thoracic and Cardiovascular Surgery, 58, 467483.

Ferrans, V J, Spray, T L, Billingham, M E, and $\frac{9}{9}$ Roberts, W C (1978). Structural changes in glu- $\rightarrow$ taraldehyde-treated porcine heterografts used as substitute cardiac valves. American Journal of Cardiology, 41, 1159-1184.

Fishbein, M C, Gissen, S A, Collins, J J jun, Bar- N samian, E M, and Cohn, L H (1977). Pathologic N findings after cardiac valve replacement with glu- $\omega$ taraldehyde-fixed porcine valves. American Journal of Cardiology, 40, 331-337.

Ionescu, M I, Tandon, A P, Mary, D A S, and Abid, A (1977). Heart valve replacement with the Ionescu-? Shiley pericardial xenograft. Journal of Thoracic $\square$ and Cardiovascular Surgery, 73, 31-42.

Johnson, A, Thompson, S, Vieweg, W V R, Daily, $\mathbb{D}$ P O, Oury, J, Tsuji, J, and Peterson, K (1976). In $\mathbb{\Phi}$ vivo function of the stent-mounted porcine heterograft in the aortic position. Circulation, 54, supnl 2, abstract No 0576. 


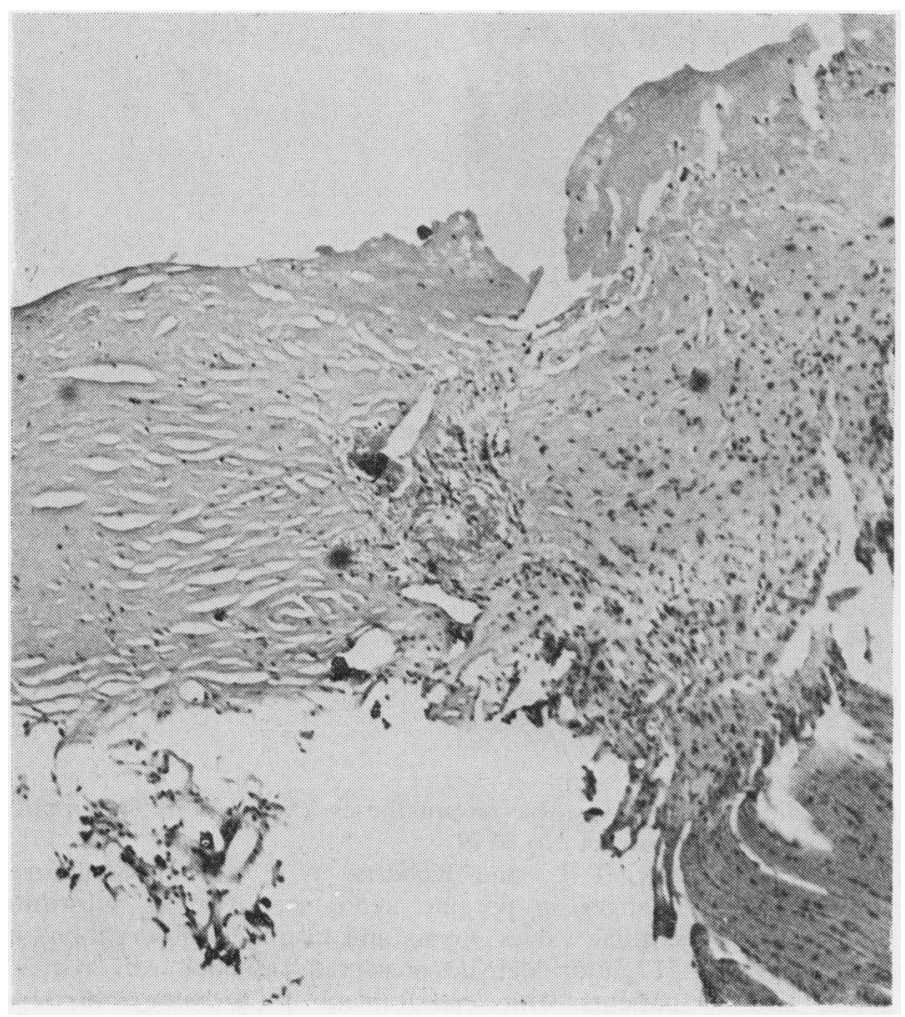

Fig 5 Photomicrograph of junction between host tissue and implanted valve six months after operation showing no ingrowth of host cellular elements into implanted valve (left half of photomicrograph). These surfaces are also devoid of host fibrous tissue $(H \& E \times 80)$.

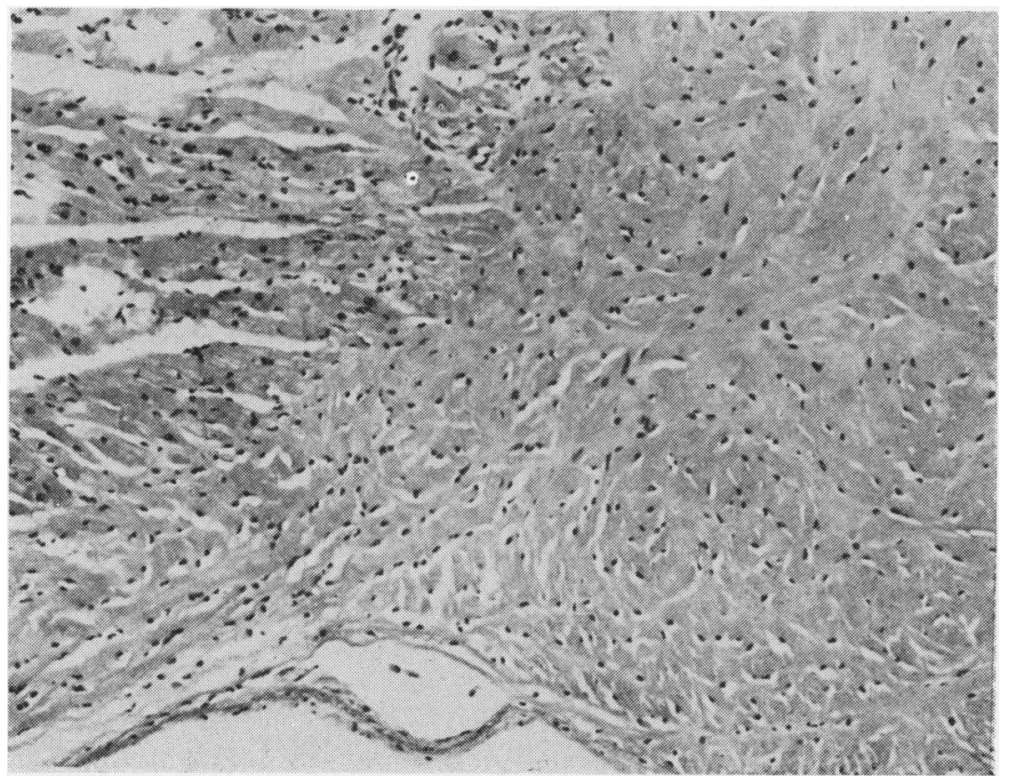

Fig 6 Right side of this photomicrograph shows retention of cellular elements of donor tissue. Only occasional foci of disarray of collagen tissue are noted $(H \& E \times 128)$. 


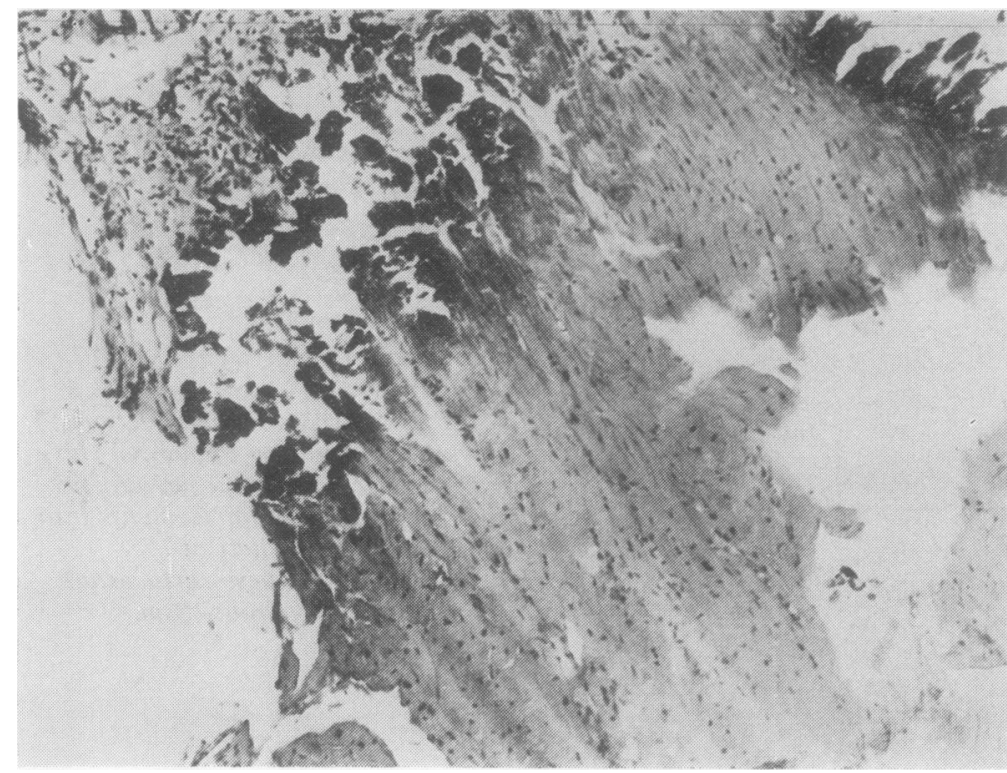

Fig 7 Photomicrograph of a pulmonary valve six months after implantation. Severe distortion of normal architecture, necrosis, and some foci of calcification $(H \& E \times 80)$.

Lendrum, A C, Fraser, D S, Slidders, W, and Henderson, $R$ (1962). Studies on the character and staining of fibrin. Journal of Clinical Pathology, 15, 401-413. Lurie, A J, Miller, R R, Maxwell, K, Vismara, L A, Hurley, E J, and Mason, D T (1976). Postoperative hemodynamic assessment of the glutaraldehydepreserved porcine heterograft in the aortic and mitral positions. Circulation, 54, suppl 2, abstract No 0577.

Oyer, P E, Stinson, E B, Griepp, R B, Shumway, N E (1977). Valve replacement with Starr-Edwards and Hancock prostheses. Annals of Surgery, 186, 301309.

Spray, T L, Arnett, E N, Morrow, A G, and Roberts, W C (1976). Structural changes in porcine xenograft cardiac valve bioprostheses. Circulation, 54, suppl 2, abstract No 0579.

Spray, T L, and Roberts, W C (1977). Structural changes in porcine xenografts used as substitute cardiac valves. Gross and histologic observations in $\mathbb{Q}$ 51 glutaraldehyde preserved Hancock valves in $41 \stackrel{\circ}{\rightarrow}$ patients. American Journal of Cardiology, 40, 319-음 330.

Zuhdi, N (1976). The porcine aortic valve bioprosthesis: a significant alternative. Annals of Thoracic Surgery, 21, 573-575.

Requests for reprints to: Dr Endre Bodnar, Department of Surgery, Cardiothoracic Institute, 2 Beaumont 0 Street, London W1N 2DX. 